\title{
Experimental Evaluation of a Control System for an Absolute Angle Measuring Micromachined Gyroscope
}

\author{
Chris Painter \\ BAE SYSTEMS \\ Westlake Village, CA 91362 \\ Email: chris.painter@baesystems.com
}

\author{
Andrei Shkel \\ Dept. of Mechanical and Aerospace Engineering \\ University of California, Irvine \\ Irvine, CA 92697 \\ Email: ashkel@uci.edu
}

\begin{abstract}
This work presents the experimental implementation and evaluation of a feedback control system for compensation of energy dissipation and suppression of anisoelasticity based errors in a micromachined absolute angle measuring gyroscope. The control system was implemented with a high speed, real time DSP board using MATLAB Simulink code downloaded onto the board. A single crystal silicon prototype was fabricated and used as a test bed. Experimental results demonstrated that the control system maintains the gyroscope motion at a defined energy level with some steady state error while simultaneously reducing anisoelasticity induced angular momentum by $31 \%$.
\end{abstract}

\section{INTRODUCTION}

Micromachined vibratory gyroscopes are currently finding their way into both automotive and short term tactical grade applications. However, the levels of performance fall short of the requirements for navigation grade applications [1]. One of the challenges is that all commercially available micromachined gyroscopes are angular rate measuring devices, whereas navigation applications typically require absolute angle measurements. This places a tremendous performance requirement on rate measuring gyroscope performance where angular rates, along with measurement errors, are electronically integrated to determine angle. As a potential solution, a micromachined gyroscope capable of directly measuring absolute angles of rotation was recently proposed [2].

The challenge is that inherent in all micromachined gyroscopes are energy dissipation mechanisms and structural imperfections. Both of these effects prevent the successful implementation of an angle measuring gyroscope and must be compensated for without disrupting the angle measuring capabilities of the device. In this work, a previously designed feedback control system [3] which fulfills this requirement is implemented on a high speed DSP board and evaluated on a bulk micromachined gyroscope test bed. The gyroscope consists of a $100 \mu m$ thick single mass structure suspended above the substrate using folded beam suspension flexures and uses electrostatic parallel plate electrodes for both actuation and sensing (Figure 1).

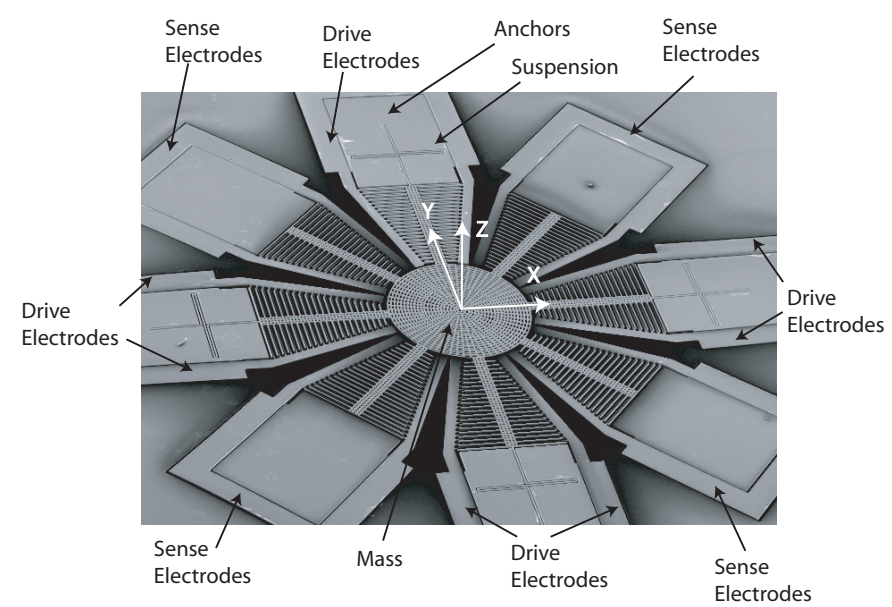

(a)

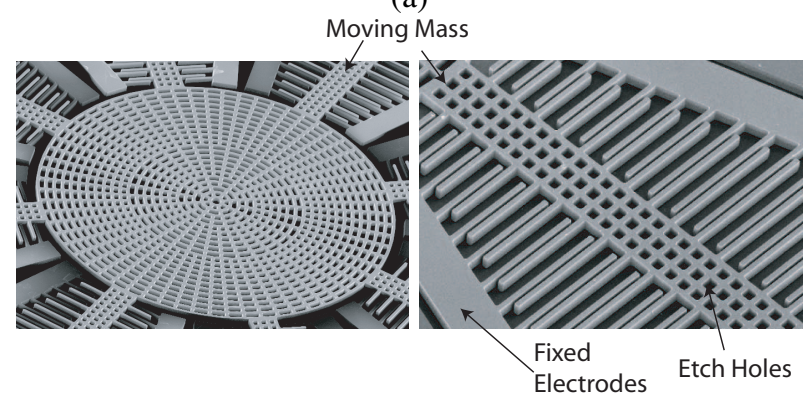

(b)

Fig. 1. (a) The gyroscope test bed consists of a $100 \mu m$ thick mass suspended using a folded beam suspension. (b) Electrostatic parallel plates are used for both driving and sensing of the device.

\section{GYROSCOPE OPERATION AND ERRORS}

Because the bulk micromachined gyroscope has a high stiffness along the $\mathrm{Z}$ direction, the dynamic model can be approximated as a two degree of freedom mass-spring-damper in the X-Y plane (Figure 2a). Further, assuming that the two natural frequencies of the primary operational modes are much greater than the input angular rate and the input angular rate is constant $(\dot{\Omega}=0)$, the general linear dynamics of the gyroscope 


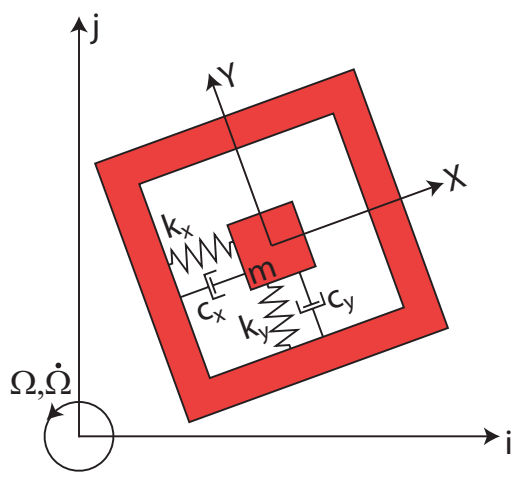

(a)

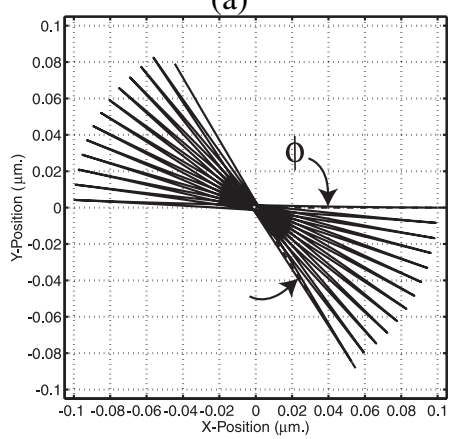

(b)

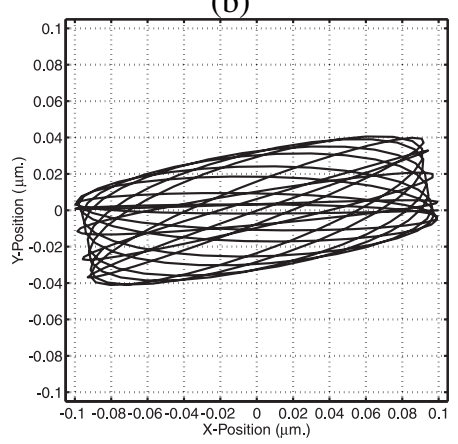

(c)

Fig. 2. (a) Two degree of freedom vibratory mass-spring-damper model of the gyroscope. (b) Under ideal operation where damping is zero and the suspension has isotropic stiffness $\left(k_{x}=k_{y}=k\right)$, the line oscillation precesses by angle $\phi$ in the presence of an input angular rate $\Omega$ where $\phi=$ $-\int \Omega d t$ (c) With a non-isotropic stiffness, the oscillation locks to principal stiffness axis and develops elliptical trajectories proportional to the split in the eigenfrequencies.

can be approximated as

$$
\begin{aligned}
m \ddot{x}+k_{x} x & =-k_{x y} y-c_{x} \dot{x} \\
m \ddot{y}+k_{y} y & =-k_{x y} x-c_{y} \dot{y}
\end{aligned}
$$

Here, $k_{x y}$ represents stiffness coupling between the $X$ and $Y$ axes. Under ideal operation, terms on the right hand side of the equation are zero and the stiffnesses along the operational axes are equal $\left(k_{x}=k_{y}=k\right)$. Assuming the device is initially vibrating along an arbitrary axis ( $X$ in this case), an input angular velocity will cause precession of the vibration pattern by angle $\phi$ (Figure 2b). It can be shown [4] that the precession angle is directly equal to the negative angle of rotation $(\phi=$ $-\int \Omega d t$, which can be calculated directly from the position and velocity of the mass by

$$
\tan 2 \phi=\frac{2\left(\omega_{n}^{2} x y+\dot{x} \dot{y}\right)}{\omega_{n}^{2}\left(x^{2}-y^{2}\right)+\left(\dot{x}^{2}-\dot{y}^{2}\right)}
$$

where $\omega_{n}^{2}=k / m$. Under realistic operation (Equation 1), damping will cause dissipation of the oscillation amplitude, eventually reducing it to zero. Even if the damping is compensated for, small variations and coupling in the stiffness will cause the oscillation pattern to lock to a principal axis of elasticity and develop into elliptical Lissajous figures (Figure 2c). Variations in the stiffness which arise during fabrication and testing under ideal laboratory conditions can potentially be compensated for using some type of trimming technique. However, variations can arise in-run that could not have been anticipated. As even minute changes in the stiffness, due to thermal stresses and electrostatic nonlinearities for example, can render the angle gyroscope inoperable, feedback control will always be necessary.

\section{FEedBACK CONTROL System}

An important consideration in the feedback control design is that the control must simultaneously reduce errors, while not interfering with the angle measurments. The control system implemented in this work was first analytically presented for micromachined angle measuring gyroscopes in [3], and the proof of non-interference of this control system topology has been done in several publications [4], [5]. The first portion of the control system is for damping compensation and has the form

$$
\left\{\begin{array}{l}
F_{x} \\
F_{y}
\end{array}\right\}=-\gamma_{2} \cdot \Delta E \cdot\left\{\begin{array}{l}
\dot{x} \\
\dot{y}
\end{array}\right\}
$$

where $F_{x}$ and $F_{y}$ are applied feedback forces. Here, $\gamma_{2}$ is a constant gain, $\dot{x}$ and $\dot{y}$ are the velocities along the $X$ and $Y$ directions, and $\Delta E$ is the variation in instantaneous system energy, given as

$$
\Delta E=E_{0}-\frac{\omega_{n}^{2}\left(x^{2}+y^{2}\right)+\left(\dot{x}^{2}+\dot{y}^{2}\right)}{2}
$$

where $E_{0}$ denotes a prescribed nominal reference energy normalized with respect to the effective mass. To compensate for stiffness variations and coupling, a control law which suppresses the angular momentum $H$ is implemented

$$
\left\{\begin{array}{l}
F_{x} \\
F_{y}
\end{array}\right\}=-\gamma_{1} \cdot H \cdot S^{T} \cdot\left\{\begin{array}{l}
x \\
y
\end{array}\right\}
$$

where $\gamma_{1}$ is a constant gain and $S$ is a $2 \times 2$ skew symmetric matrix. The complexity of these control systems makes them challenging to implement in a strictly analog domain. For this reason, a digital control system was used.

\section{FABRiCATION AND CONTROL DESIGN}

The following sections describe the fabrication process used to design the sensor and the digital implementation of the control. 
TABLE I

GYROSCOPE PROTOTYPE PARAMETERS

\begin{tabular}{|c|c|}
\hline Surface Area & $1.47 \times 10^{-6} \mathrm{~m}^{2}$ \\
\hline Thickness & $100 \times 10^{-6} \mathrm{~m}$ \\
\hline Mass & $3.42 \times 10^{-7} \mathrm{~kg}$ \\
\hline Suspension Width & $10.0 \times 10^{-6} \mathrm{~m}$ \\
\hline Theoretical Stiffness & $201 \mathrm{~N} / \mathrm{m}$ \\
\hline Measured In-Plane Frequency & $2.7 \mathrm{kHz}$ \\
\hline Parallel Plate Gap Spacing & $10 \times 10^{-6} \mathrm{~m}$ \\
\hline Parallel Plate Overlap Area & $5.04 \times 10^{-7} \mathrm{~m}^{2}$ \\
\hline
\end{tabular}

\section{A. Fabrication}

A bulk micromachining process was used in the fabrication, which was conducted at UCI's INRF facility. To summarize the process, a photoresist pattern was developed on top of a silicon-on-insulator (SOI) wafer consisting of a $100 \mu \mathrm{m}$ thick conductive single crystal silicon device layer, followed by a 4 $\mu \mathrm{m}$ thick silicon dioxide layer which served as the insulator between the device layer and the $500 \mu \mathrm{m}$ conductive single crystal silicon handle layer. The SOI wafer was then etched in a deep reactive ion etcher (DRIE) for 1.5 hours through the top silicon layer down to the oxide to define the features. The wafer was diced and the sample was then placed in an HF bath for 25 minutes to release the mass. The sample was then mounted in a DIP package, wire bonded, and connected to external analog preamplifiers. The structural and electronic parameters of the gyroscope are shown in Table I.

\section{B. Digital Control Implementation}

The hardware used in the characterization was the DS1103 control system from dSPACE [6]. Realistically, the system was found to be capable of supporting a sophisticated control algorithm using 2 ADC inputs and 3 DAC outputs while running in real time at a sampling rate of $10 \mu \mathrm{s}$. The output from the processor connects to a breakout board with BNC posts that transmit the signals to the gyroscope. The code for implementing the control on the dSPACE board was written in Simulink and is shown in part in Figure 3. The connections of the device were configured as shown in Figure 4, where $V_{45 / 225}$ and $V_{315 / 135}$ are the sense voltage inputs into the dSPACE board. The carrier $V_{c}$ of $8 \mathrm{~V}$ at $20 \mathrm{kHz}$ was produced by the dSPACE board and was sent to a DC power supply, which added a DC bias $V_{D C}=50 \mathrm{~V}$ before the signal was applied to the mass. Voltages $V_{x}$ and $V_{y}$ were used for actuating the structure and were output directly from 2 of the DAC channels on the dSPACE board onto the drive electrodes located at $0^{\circ}$ and $90^{\circ}$ on the gyroscope, respectively. The digital control consists of a demodulator, followed by a state transformation which maps the sensed positions and velocities from the sense directions (located at $45^{\circ}$ to the $X$ and $Y$ directions) to the $X$ and $Y$ directions. This signal is then input into blocks using the feedback control topologies of Equations (3) and (5).

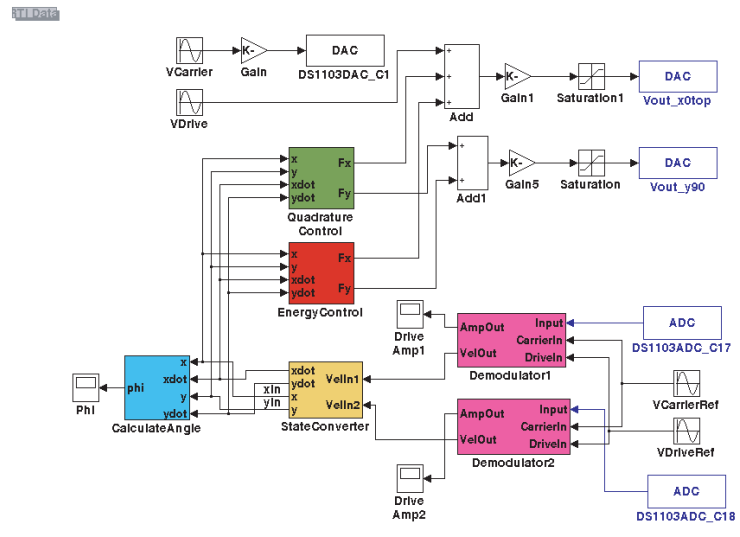

Fig. 3. Simulink code used in the digital control implementation

\section{EXPERIMENTAL RESULTS}

The energy compensating control system was first activated, with the desired mass normalized energy level $E_{0}$ set to 500 $J / k g$. This energy level corresponds to the actual mechanical energy of the gyroscope times the mechanical to electrical gain through the preamplifiers. The controller drove the oscillation to a steady motion about the first principal axis of elasticity, oriented at an angle $12^{\circ}$ from the $X$ axis (Figure 5a), a behavior which was also observed in prior computer simulations [7]. Additionally, there was an observed steady state error of 160 $J / k g$ between the desired and actual energy levels, as well as high and low frequency variations in the energy amplitude (Figure 5b). These errors arise in part from variation in the actual natural frequency of the gyroscope from the estimated natural frequency in the calculation of energy from Equation (4), which is hard coded in the control system.

With no quadrature control, there was observed ellipticity in the trajectory of the device (Figure 6a). The mass normalized angular momentum related to the ellipticity was calculated in the real time control to be $2.1 \times 10^{-3} \mathrm{~m}^{2} / \mathrm{s}$ (Figure 6c). The quadrature control system was then activated with the energy compensating control still running and the ellipticity was noticeably reduced (Figure 6b). This was also confirmed quantitatively through reduction in the angular momentum by $31 \%$ (Figure 6d).

\section{CONCLUSION}

In this paper, we have presented the digital implementation of an energy dissipation and structural imperfection compensating control for maintaining the ideal motion of an angle measuring gyroscope. While the energy compensating control was demonstrated to maintain the motion of the mass, low and high frequency variations in the calculated energy were observed. These errors were due in part to the difference between the actual natural frequency and the hard coded natural frequency in the dSPACE control block. The addition of the quadrature control successfully reduced the angular momentum by a factor of $31 \%$, verifying the analytical and computer simulations used to develop the control algorithms. 


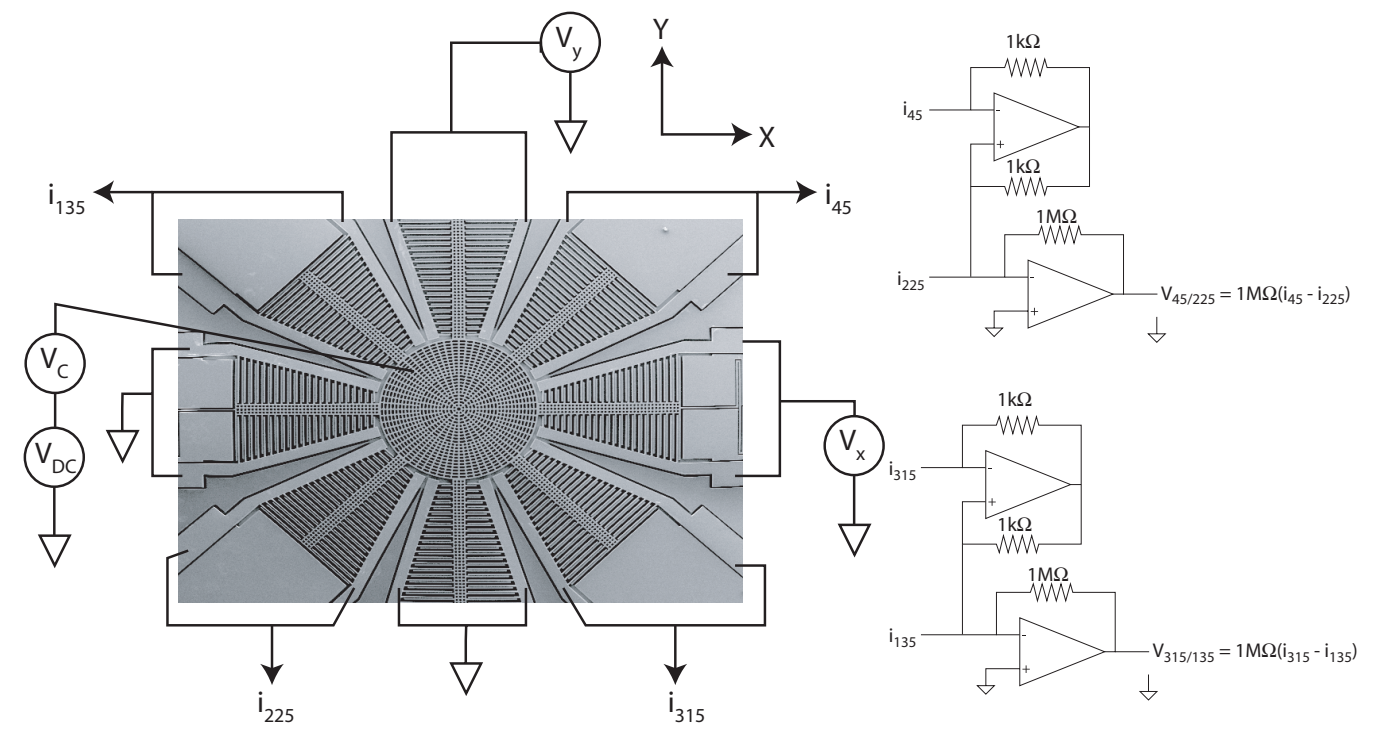

Fig. 4. Analog connections to the gyroscope prototype

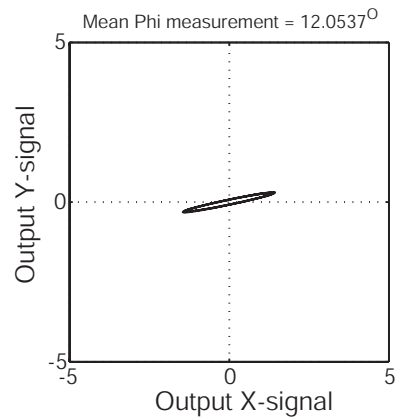

(a)

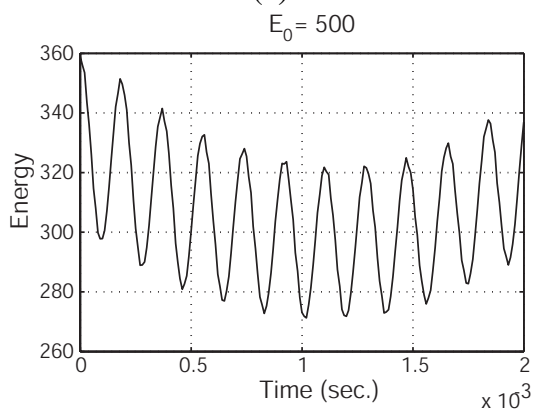

(b)

Fig. 5. (a) The energy controller drove the device to steady state oscillation along the principal axis with the weakest stiffness. (b) It was also observed that the energy was not constant, but varied according to a low and high frequency component.

\section{REFERENCES}

[1] N. Barbour and G. Schmidt, "Inertial sensor technology trends," Sensors and Actuators A, vol. 1, no. 4, pp. 332-339, December 2001.

[2] A. Shkel and R. Howe, "Micro-machined angle-measuring gyroscope," November 2002, U.S. Patent 6,481,285.

[3] A. Shkel, R. Horowitz, A. Seshia, and R. Howe, "Dynamics and control of micromachined gyroscopes," in The American Control Conference, San Diego, CA, June 1999, pp. 2119-2124.

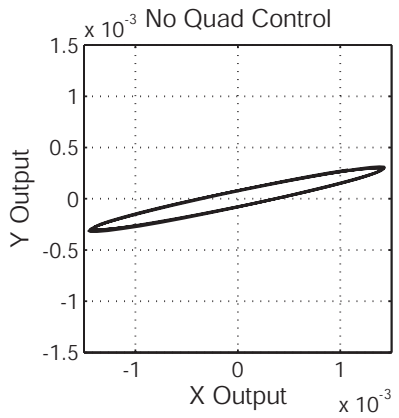

(a)

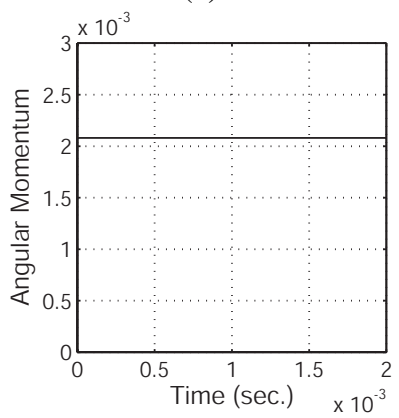

(c)

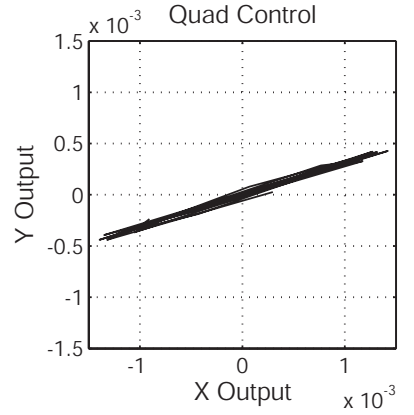

(b)

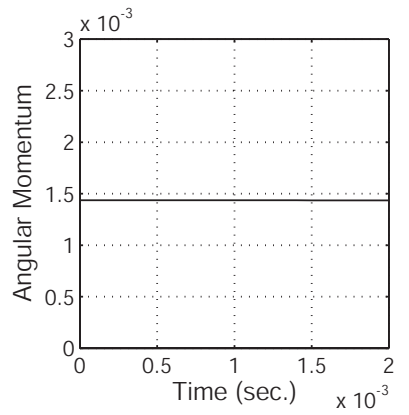

(d)
Fig. 6. (a) With no quadrature control, there is ellipticity, due in part to anisoelasticity. (b) With the addition of the quadrature control, ellipticity is reduced. (c) Without the control, the angular momentum is $2.1 \times 10^{-3} \mathrm{~m}^{2} / \mathrm{s}$. (d) With the control, there is a $31 \%$ reduction in angular momentum.

[4] B. Friedland and M. Hutton, "Theory and error analysis of vibratingmember gyroscope," IEEE Transactions on Automatic Control, vol. AC23, no. 4, pp. 545-556, 1978.

[5] V. Zhuravlev, "Oscillation shape control in resonant systems," J. Appl. Maths Mechs., vol. 56, no. 5, pp. 725-735, 1992.

[6] http://www.dspace.com.

[7] C. Painter and A. Shkel, "Active structural error suppression in MEMS vibratory rate integrating gyroscopes," IEEE Sensors Journal, vol. 3, no. 5, pp. 595-606, October 2003. 